\title{
Rastreio do cancro do colo do útero em mulheres homossexuais: que evidência?
}

Alice Jeri, ${ }^{1}$ Ana Bessa Monteiro²

\section{RESUMO}

Objetivo: Rever a evidência sobre a relevância de rastrear para o cancro do colo do útero (CCU) mulheres saudáveis com comportamento homossexual, tendo em conta o risco da neoplasia nesta população.

Fontes de dados: Bases de dados MEDLINE, National Guideline Clearinghouse, National Institute for Health and Care Excellence, Canadian Medical Association Practice Guidelines Infobase, Cochrane Library, Bandolier, DARE e Trip Database.

Métodos de Revisão: Revisão baseada na evidência de artigos publicados nos últimos cinco anos (de 1 de outubro de 2011 a 30 de setembro de 2016), nas línguas inglesa, espanhola e portuguesa. Foram utilizadas combinações dos termos MESH: female homosexuality, mass screening, diagnosis, uterine cervical neoplasms e papillomaviridae, e o termo livre cervical cancer. Para avaliação do Nível de Evidência (NE) e de Força de Recomendação (FR) foi utilizada a escala Strength of Recommendation Taxonomy (SORT), da American Family Physician.

Resultados: Obtiveram-se 88 artigos, dos quais seis cumpriam os critérios de inclusão: cinco normas de orientação clínica (uma com FR A, duas com FR B e duas com FR C) e um estudo de coorte prospetivo (NE 3). As normas internacionais apresentam algumas limitações e inconsistências relativamente à indicação do rastreio nas mulheres homossexuais, recomendando a maioria a sua inclusão. $O$ estudo coorte encontrou alterações em citologias e testes de Papilomavírus Humano (HPV) de mulheres com comportamento homossexual, colocando-as em risco de desenvolver CCU.

Conclusões: Mulheres com comportamento homossexual podem estar em risco de infeção pelo HPV e de desenvolvimento de CCU, devendo assim ser incluídas no rastreio populacional com a mesma periodicidade das restantes mulheres (FR B). São, contudo, necessários mais estudos que reforcem esta evidência. Dada a menor sensibilização deste grupo quanto ao benefício do rastreio sugere-se a implementação de medidas educativas dirigidas, visando aumentar a adesão. Sugere-se ainda revisão das normas nacionais, clarificando a indicação do rastreio nesta população.

Palavras-chave: Programa de rastreamento; Diagnóstico; Neoplasia do colo do útero; Homossexualidade feminina; Comportamento sexual.

\section{INTRODUÇÃO}

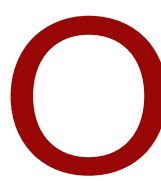

cancro do colo do útero (CCU) ocupa o terceiro lugar na prevalência e o quarto nas causas de morte por cancro, entre mulheres, em todo o mundo. ${ }^{1-2}$ É o $9^{\circ}$ cancro mais frequente nas mulheres portuguesas, com uma incidência de 13,5/100.000 mulheres. ${ }^{3}$ É responsável por elevada morbilidade e mortalidade, com uma taxa padronizada de anos de vida potencial perdidos de 40,3 (por 100.000

1. Médica Interna de Medicina Geral e Familiar. USF Barão Nova Sintra, ACeS Porto Oriental

2. Médica Assistente de Medicina Geral e Familiar. USF Barão Nova Sintra, ACeS Porto Oriental mulheres abaixo dos 70 anos) em 2009, ${ }^{4}$ estabelecendo o Plano Nacional de Saúde de 2012-2016 uma meta de redução de 21,4 neste valor até ao ano de 2016 (Quadro I).

A incidência de CCU no nosso país diminuiu notoriamente nos últimos anos, em grande parte devido aos avanços conseguidos com um programa de rastreio realizado de forma oportunista ou com base populacional, dirigido a mulheres entre os 25 e os 60 anos e alicerçado na colheita de colpocitologia nos cuidados de saúde primários (CSP) ${ }^{4-5}$

Mais de $99 \%$ dos casos de CCU estão relacionados com as consequências a longo-prazo da infeção pelo Papilomavírus Humano (HPV). ${ }^{6}$ A transmissão do vírus 


\begin{tabular}{|c|c|c|c|c|c|}
\hline & \multicolumn{5}{|c|}{ VALORES } \\
\hline & $\begin{array}{c}2001 \\
\text { (a) }\end{array}$ & $\begin{array}{c}2009 \\
\text { (a) }\end{array}$ & $\begin{array}{c}\text { Projeção } \\
2016\end{array}$ & $\begin{array}{c}\text { META } \\
2016\end{array}$ & $\begin{array}{c}\text { Ganhos } \\
\text { acumulados }\end{array}$ \\
\hline $\begin{array}{l}\text { Anos de vida potenciais perdidos por tumor } \\
\text { maligno do colo do útero (/100.000 mulheres } \\
\text { abaixo dos } 70 \text { anos) }\end{array}$ & 52,4 & 40,3 & 27,0 & 18,9 & 111,4 \\
\hline
\end{tabular}

(a): ou ano mais próximo.

Adaptado de: Plano Nacional de Saúde 2012-2016. ${ }^{4}$

é feita geralmente por contacto genital, embora possa não ser a única via. ${ }^{7-8}$

Dados internacionais indicam menor adesão da população homossexual feminina ao rastreio do CCU. As causas podem incluir uma autoperceção equívoca de menor risco de infeção pelo $\mathrm{HPV}^{9}$ (sobretudo em mulheres sem coitarca com parceiro do sexo masculino); a não-identificação destas mulheres com as campanhas de promoção do rastreio, dirigidas à população em geral: ${ }^{10-11}$ bem como um afastamento relativamente aos serviços dirigidos à saúde da mulher, cujas atividades têm um importante foco no planeamento familiar e nos cuidados à mulher grávida. ${ }^{12-13}$ Desta forma, mulheres homossexuais podem apresentar risco superior de desenvolver CCU invasivo. ${ }^{14-15}$

Por seu lado, os profissionais de saúde podem igualmente questionar a relação custo-benefício do rastreio neste contexto, por subestimação do risco de CCU nesta população ou por receio de potenciais danos físicos e/ou psicológicos associados ao exame do colo uterino, nomeadamente se este resultar em rotura do hímen. ${ }^{16}$

As normas nacionais são omissas no que respeita à indicação de rastrear mulheres homossexuais. ${ }^{5,17-18} \mathrm{O}$ Programa de Rastreio de Cancro do Colo do Útero da Região Norte ${ }^{5}$ integra nos seus critérios de exclusão o não-início de atividade sexual, não definindo claramente o tipo de atividade considerada.

O presente trabalho tem como objetivo rever a evidência disponível sobre a relevância de rastrear para o CCU utentes do sexo feminino, saudáveis, com comportamento homossexual, tendo em conta o risco da neoplasia nesta população. Os autores optaram por incluir nesta revisão todas as publicações cujo conteúdo permita elucidar so- bre a atitude mais correta perante as mulheres com comportamento homossexual em geral e não apenas aquelas com comportamento homossexual exclusivo.

\section{MÉTODO}

Realizou-se uma pesquisa bibliográfica de normas de orientação clínica (NOC), revisões sistemáticas, meta-análises e estudos originais nas bases de dados MEDLINE, National Guideline Clearinghouse, National Institute for Health and Care Excellence (NICE), Canadian Medical Association Practice Guidelines Infobase, Cochrane Library, Bandolier, DARE e Trip Database de artigos publicados nos últimos cinco anos (de 1 de outubro de 2011 a 30 de setembro de 2016), nas línguas inglesa, espanhola e portuguesa. Foram utilizadas combinações dos termos MESH: female homosexuality, mass screening, diagnosis, uterine cervical neoplasms e papillomaviridae, e ainda o termo livre cervical cancer, com o objetivo de identificar o maior número de publicações relevantes, tendo em conta as assimetrias na indexação de termos entre bases de dados. Os termos foram combinados como se explicita.

Na identificação de NOC foram considerados artigos que incluíssem os termos uterine cervical neoplasms ou cervical cancer. Na pesquisa na base de dados da NICE foram utilizados os filtros guidance e accredited.

Na identificação de revisões sistemáticas e de artigos originais foram considerados os artigos que incluíssem, para além do termo female homosexuality, os termos mass screening ou diagnosis e uterine cervical neoplasms ou papillomaviridae.

A pesquisa bibliográfica foi feita no dia 1 de outubro de 2016.

Definiram-se como critérios de inclusão:

- População: mulheres homossexuais saudáveis, com idade indicada para rastreio

- Intervenção: efetuar o rastreio de CCU

- Comparação: ausência de rastreio

- Outcome: benefício expectável, tendo em conta o risco de desenvolver CCU 


\begin{tabular}{|c|c|c|}
\hline \multicolumn{3}{|r|}{ Strength of Recommendation Taxonomy, da American Family Physician } \\
\hline & Classificação & Significado \\
\hline \multirow[t]{3}{*}{ FR } & A & Recomendação baseada em evidência consistente, de boa qualidade, orientada para o doente \\
\hline & B & Recomendação baseada em evidência inconsistente, de qualidade limitada, orientada para o doente \\
\hline & $\mathrm{C}$ & $\begin{array}{l}\text { Recomendação baseada em consensos, prática clínica, opinião, orientada para a doença ou séries de casos } \\
\text { acerca do diagnóstico, tratamento prevenção ou rastreio }\end{array}$ \\
\hline \multirow[t]{3}{*}{ NE } & 1 & Estudos de boa qualidade, evidência orientada para o doente \\
\hline & 2 & Estudos de qualidade limitada, evidência orientada para o doente \\
\hline & 3 & Outra evidência \\
\hline
\end{tabular}

Legenda: FR = Força de recomendação; NE = Nível de evidência .

Foram excluídas mulheres grávidas, histerectomizadas, com antecedentes de patologia neoplásica ou displásica do colo do útero, imunossuprimidas e fora das idades indicadas para a realização do rastreio.

Para avaliação da qualidade dos estudos, atribuição de Nível de Evidência (NE) e de Força de Recomenda-

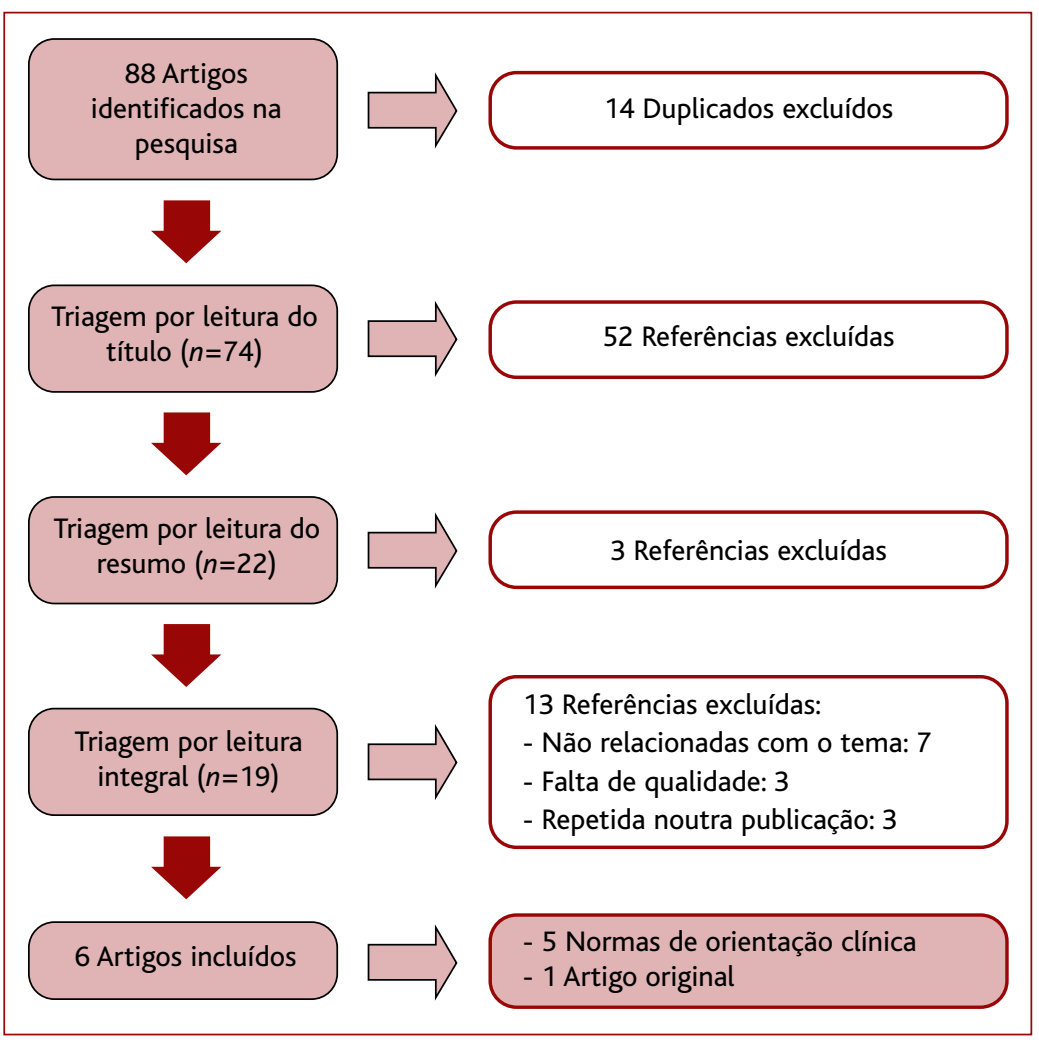

Figura 1. Fluxograma de seleção dos artigos. ção (FR) foi utilizada a escala Strength of Recommendation Taxonomy (SORT) ${ }^{19}$ da American Family Physician (Quadro II). Para avaliação da qualidade metodológica das NOC foi aplicado o Appraisal of Guidelines Research \& Evaluation Instrument (AGREE).$^{20}$

\section{RESULTADOS}

A pesquisa inicial identificou 88 artigos, dos quais se excluíram 14 por se encontrarem duplicados e 68 por não se enquadrarem no objetivo da revisão, por não cumprirem critérios de inclusão ou por não revelarem qualidade metodológica (essencialmente por pouca qualidade da informação e fraca validade interna). Foram incluídos seis artigos: cinco NOC e um estudo original. O fluxograma da seleção dos estudos encontra-se explanado na Figura 1.

As normas da Cancer Care Ontario, ${ }^{21} \mathrm{de}$ 2011, indicam que mulheres que têm sexo com outras mulheres devem seguir o mesmo regime de rastreio que as mulheres que têm sexo com homens. Do rastreio são excluídas mulheres sem atividade sexual, abrangendo esta definição a atividade vaginal/oral ou vaginal/digital. Esta norma é baseada na evidência, tendo como suporte uma revisão sistemática de elevada qualidade com opinião de peritos, ${ }^{22}$ elaborada para a versão prévia 


\section{QUADRO III. Recomendações baseadas na evidência}

Norma de Orientação Clínica

\begin{tabular}{l|l|c}
\hline Referência & \multicolumn{1}{|c|}{ Conclusões } & FR \\
\hline $\begin{array}{l}\text { Cancer Care Ontario } \\
\text { (2011) }\end{array}$ & $\begin{array}{l}\text { Mulheres que têm sexo com outras mulheres devem seguir o mesmo regime de rastreio que as } \\
\text { mulheres que têm sexo com homens. }\end{array}$ & B \\
\hline $\begin{array}{l}\text { Royal Australian College } \\
\text { of General Practitioners } \\
\text { (2012) }\end{array}$ & $\begin{array}{l}\text { Recomenda o rastreio de todas as mulheres com colo do útero e com início de atividade sexual; } \\
\text { mulheres com parceiros sexuais do sexo feminino são consideradas em risco e incluídas no } \\
\text { rastreio. }\end{array}$ & B \\
\hline $\begin{array}{l}\text { University of Michigan } \\
\text { Health System (2012) }\end{array}$ & $\begin{array}{l}\text { Mulheres sem coito vaginal não têm indicação para rastreio. } \\
\text { Noção de «coito vaginal» indefinida quanto à inclusão de práticas homossexuais. } \\
\text { Norma baseada nas recomendações da United States Preventive Services Task Force (2012), a } \\
\text { qual estende as recomendações do rastreio a todas as mulheres com colo do útero, } \\
\text { independentemente da história sexual. }\end{array}$ & A \\
\hline $\begin{array}{l}\text { Toward Optimized } \\
\text { Practice Cervical Cancer } \\
\text { Screening Working Group } \\
\text { (2016) }\end{array}$ & $\begin{array}{l}\text { Inclui no rastreio mulheres que iniciaram atividade sexual, incluindo penetração ou atividade } \\
\text { sexual oral ou digital envolvendo a área genital com parceiro de qualquer género. }\end{array}$ & C \\
\hline $\begin{array}{l}\text { British Columbia (2016) } \\
\text { Recomenda o rastreio de mulheres com relações homossexuais. }\end{array}$ & C
\end{tabular}

da norma, a qual atribuiu uma FR B à recomendação referida (Quadro III).

As normas da Royal Australian College of General Practitioners, ${ }^{23}$ de 2012, recomendam o rastreio a todas as mulheres com colo do útero e que iniciaram atividade sexual; mulheres com parceiros sexuais do sexo feminino são consideradas em risco e incluídas no rastreio. Estas recomendações são classificadas com FR B (Quadro 3). Esta norma é baseada na evidência, tendo como suporte as recomendações emitidas pelo National Health and Medical Research Council (Austrália), ${ }^{24}$ elaboradas com base numa revisão sistemática de elevada qualidade, bem como na opinião de peritos.

As orientações da University of Michigan Health Sys$t e m,{ }^{25}$ de 2012, não indicam o rastreio em mulheres sem coito vaginal, considerando que não apresentam risco de desenvolver CCU. No entanto, não é definida a noção de «coito vaginal», não sendo clara quanto à inclusão de práticas homossexuais com eventual penetração vaginal. Na bibliografia que suporta o documento encontra-se a norma de orientação clínica da United States Preventive Services Task Force (2012), ${ }^{26}$ a qual estende as recomendações do rastreio a todas as mulheres com colo do útero, independentemente da história sexual (desde que nas idades recomendadas), atribuindo a esta recomendação uma FR A (Quadro III).

As recomendações do Toward Optimized Practice Cervical Cancer Screening Working Group, ${ }^{27}$ de 2016, são consonantes em incluir no rastreio mulheres que iniciaram atividade sexual, incluindo nesta noção a penetração, bem como atividade sexual oral ou digital envolvendo a área genital com um parceiro de qualquer género. A estas normas atribui-se FR C por se basearem numa meta-análise de boa qualidade, embora orientada para a doença (Quadro III).

As normas da British Columbia, ${ }^{28} \mathrm{de} 2016$, recomendam também o rastreio de mulheres com relações homossexuais, tendo por base bibliografia semelhante à da norma anterior, pelo que se atribui FR C (Quadro III).

O estudo original, multicêntrico, de seguimento de uma coorte prospetiva, publicado por Stewart Massad e colaboradores em janeiro de 2015 (Quadro IV), acompanhou 3.766 mulheres norte-americanas, seropositivas para o vírus da imunodeficiência humana (VIH) ou com risco de infeção, tendo como objetivo determinar a frequência de citologias anormais (segundo o sistema de Bethesda) e positividade em testes de HPV. As mulheres foram examinadas a cada seis meses, entre outubro de 1994 e outubro de 2010. Das mulheres incluídas, 2.791 eram seropositivas para VIH e 975 sero- 
QUADRO IV. Análise comparativa de ambos os grupos de inquiridos

Estudo coorte prospetivo

\begin{tabular}{|l|l|l|c}
\hline Referência & \multicolumn{1}{|c|}{ População } & \multicolumn{1}{c|}{ Conclusões } & NE \\
\hline $\begin{array}{l}\text { Stewart Massad } \\
\text { et al (2015) }\end{array}$ & $\begin{array}{l}n=3.766 \text { (VIH + e } \\
\text { seronegativas) } \\
\text { Incluídas 975 mulheres } \\
\text { seronegativas para VIH }\end{array}$ & $\begin{array}{l}\text { Mulheres com sexo com mulheres não infetadas com VIH devem ser } \\
\text { rastreadas para CCU da mesma forma que mulheres com sexo com homens, } \\
\text { de acordo com as normas para a população geral. }\end{array}$ & 3 \\
\end{tabular}

Legenda: NE = Nível de evidência; VIH = Vírus da imunodeficiência humana; VIH+ = Seropositivas para o vírus da imunodeficiência humana.

negativas. Destas, 120 tinham tido sexo com homem nos últimos cinco anos e nenhuma relação com mulheres neste período ou durante o estudo (mulheres com sexo com homens); 24 relataram ter tido sexo com uma mulher nos últimos cinco anos e ausência de relacionamento com parceiro do sexo masculino no mesmo período (mulheres com sexo com mulheres), sendo para o presente trabalho considerado apenas este último grupo. A definição de sexo incluiu penetração vaginal ou anal, ou sexo oral.

Nas mulheres seronegativas, a duração média do follow-up foi de 8,3 anos. Das 369 citologias realizadas a mulheres com sexo com mulheres seronegativas, 23 tiveram resultados anormais $(6,2 \%)$; destas, uma teve resultado correspondente a lesão intraepitelial escamosa de alto grau ou superior. Do total dos 168 testes de HPV adequados realizados a mulheres com sexo com mulheres seronegativas, em $21(12,5 \%)$ foi detetado HPV de qualquer tipo e em oito de tipo oncogénico $(4,8 \%)$.

O estudo conclui que mulheres com sexo com mulheres não infetadas com VIH devem ser rastreadas para CCU da mesma forma que mulheres com sexo com homens, de acordo com as guidelines vigentes para a população geral.

Como limitações refere-se o número relativamente limitado de mulheres com sexo com mulheres estudado; o não esclarecimento quanto à autoclassificação da orientação sexual das participantes; o não registo de informação quanto a fatores que podem influenciar o risco de HPV e o surgimento de anormalidades na citologia, incluindo exposição a parceiros masculinos num período superior aos cinco anos prévios ao estudo, número de parceiros sexuais e práticas sexuais específicas.
Refere-se ainda que o rastreio regular das participantes no estudo contrasta com a baixa frequência de rastreio observada em geral nas mulheres com sexo com mulheres, as quais podem apresentar maior risco de anormalidades na CCV a longo prazo. Considerando tratar-se de um estudo orientado para a doença e, apesar da favorável qualidade metodológica, atribui-se NE 3.

\section{DISCUSSÃO/CONCLUSÕES}

A evidência indica que as mulheres com comportamento homossexual podem estar em risco de contrair infeção pelo HPV e de desenvolver CCU, devendo assim ser incluídas no rastreio de base populacional com a mesma periodicidade das restantes mulheres (FR B).

As vias para infeção pelo HPV podem incluir o contacto vaginal/oral, vaginal/digital, a partilha de objetos sexuais ou mesmo a transmissão através de fômites contaminados. ${ }^{7}$ Refere-se a relevância da história sexual prévia tanto das mulheres em causa como das suas parceiras, nomeadamente no que concerne a práticas heterossexuais prévias, a qual pode, contudo, não ser revelada ou valorizada pelas utentes.

As autoras realçam que as normas de orientação clínica não apresentam suficiente robustez nesta matéria e que o estudo incluído é de natureza observacional e orientado para a doença (diagnóstico de infeção por HPV ou de anormalidades citológicas). Os estudos na base desta evidência podem estar sujeitos a eventuais vieses de seleção, devido à dificuldade em obter uma amostra aleatória de utentes homossexuais (tendo em conta a natureza voluntária da participação); e a vieses de informação, incluindo viés de desejabilidade social, dificultando a classificação dos comportamentos sexuais das utentes. 
Não se encontraram estudos experimentais ou estudos observacionais orientados para o doente que quantifiquem os benefícios expectáveis a longo prazo com a consolidação do rastreio nesta população específica, em termos de redução da morbimortalidade. Esta situação pode assentar em limitações éticas quanto à experimentação que implique excluir do rastreio do CCU mulheres que potencialmente beneficiariam dele, bem como de limitações quanto ao recrutamento de participantes (considerando a natureza privada e frequentemente encoberta dos comportamentos sexuais), limitando a realização de estudos observacionais robustos e abrangentes, capazes de relacionar a adesão ao rastreio com resultados na melhoria do estado de saúde.

Adicionalmente, a maioria dos sistemas de saúde não permite a identificação da orientação ou comportamento sexual como critério demográfico, limitando o uso dos registos médicos para fins de investigação. Outra limitação prende-se com a indefinição das práticas sexuais incluídas em algumas NOC e estudos, nomeadamente com a não-definição do conceito de «virgindade» ou «coito vaginal».

É também de referir a inexistência de avaliação de danos potencialmente associados ao exame ginecológico de mulheres sem coito vaginal prévio, designadamente danos físicos, psicológicos e sociais relacionados com a eventual rotura do hímen (possível mesmo com a utilização de espéculos de menor dimensão), assente em valores culturais subjetivos e de difícil avaliação.

A necessidade de evidência mais robusta, associada ao importante potencial de melhoria na prática clínica, indica a realização de estudos mais abrangentes, orientados para o doente e com desenho metodológico que possibilite minimizar potenciais vieses e analisar eventuais danos, permitindo esclarecer o balanço custo-benefício de incluir mulheres homossexuais exclusivas no rastreio do CCU.

Não obstante, tendo em conta os benefícios expectáveis com o alargamento do rastreio às mulheres com comportamentos homossexuais e a menor sensibilização deste grupo quanto à sua indicação, sugere-se a implementação de medidas de educação dirigidas a utentes e profissionais visando aumentar a sua adesão. Sugere-se ainda revisão das normas nacionais, clarificando a indicação do rastreio nesta população.

\section{REFERÊNCIAS BIBLIOGRÁFICAS}

1. Jemal A, Bray F, Center MM, Ferlay J, Ward E, Forman D. Global cancer statistics. CA Cancer J Clin. 2011;61(2):69-90.

2. Jemal A, Bray F, Center MM, Ferlay J, Ward E, Forman D. Global cancer statistics. CA Cancer J Clin. 2011;61(2):69-90.

3. Direção-Geral da Saúde. Portugal - Doenças oncológicas em números 2015: programa nacional para as doenças oncológicas. Lisboa: DGS; 2016.

4. Direção-Geral da Saúde. Plano nacional de saúde 2012-2016. Lisboa: DGS; 2013.

5. Administração Regional de Saúde do Norte. Programa de rastreio do cancro do colo do útero na Região Norte. Porto: ARS Norte; 2009.

6. Walboomers JM, Jacobs MV, Manos MM, Bosch FX, Kummer JA, Shah $\mathrm{KV}$, et al. Human papillomavirus is a necessary cause of invasive cervical cancer worldwide. J Pathol. 1999;189(1):12-9.

7. Ferenczy A, Bergeron C, Richart RM. Human papillomavirus DNA in fomites on objects used for the management of patients with genital human papillomavirus infections. Obstet Gynecol. 1989;74(6):950-4.

8. Strauss S, Sastry P, Sonnex C, Edwards S, Gray J. Contamination of environmental surfaces by genital human papillomaviruses. Sex Transm Infect. 2002;78(2):135-8.

9. Eaton L, Kalichman S, Cain D, Cherry C, Pope H, Fuhrel A, et al. Perceived prevalence and risks for human papillomavirus (HPV) infection among women who have sex with women. JWomens Health (Larchmt). 2008; 17(1):75-83.

10. Brown A, Hassard J, Fernbach M, Szabo E, Wakefield M. Lesbians' experiences of cervical cancer. Health Promot J Aust. 2003;14(2):128-32.

11. McIntyre L, Szewchuk A, Munro J. Inclusion and exclusion in midlife lesbians' experiences of the Pap test. Cult Health Sex. 2010;12(8): 885-98.

12. Cochran SD, Mays VM, Bowen D, Gage S, Bybee D, Roberts SJ, et al. Cancer-related risk indicators and preventive screening behaviors among lesbians and bisexual women. Am J Public Health. 2001;91(4):591-7.

13. Tracy JK, Lydecker AD, Ireland L. Barriers to cervical cancer screening among lesbians. JWomens Health (Larchmt). 2010;19(2):229-37.

14. Frega A, Cenci M, Stentella P, Cipriano L, De loris A, Alderisio M, et al. Human papillomavirus in virgins and behaviour at risk. Cancer Lett. 2003;194(1):21-4.

15. Doerfler D, Bernhaus A, Kottmel A, Sam C, Koelle D, Joura EA. Human papilloma virus infection prior to coitarche. Am J Obstet Gynecol. 2009;200(5):487.e1-e5.

16. Marrazzo JM. Genital human papillomavirus infection in women who have sex with women: a concern for patients and providers. AIDS Patient Care STDS. 2000;14(8):447-51.

17. Resolução do Conselho de Ministros n. ${ }^{\circ}$ 129/2001, de 17 de agosto. Diário da República. $1^{\text {a }}$ Série(190).

18. Coordenação Nacional para as Doenças Oncológicas. Plano nacional de prevenção e controle das doenças oncológicas 2007-2010: programa de desenvolvimento. Lisboa: Ministério da Saúde; 2009.

19. Ebell MH, Siwek J, Weiss BD, Woolf SH, Susman J, Ewigman B, et al. Strength of Recommendation Taxonomy (SORT): a patient-centered approach to grading evidence in the medical literature. Am Fam Physician. 2004;69(3):548-56.

20. AGREE Collaboration. Development and validation of an international 
appraisal instrument for assessing the quality of clinical practice guidelines: the AGREE project. Qual Saf Health Care. 2003;12(1):18-23.

21. Murphy J, Kennedy E, Dunn S, Fung Kee Fung M, Gzik D, McLachlin CM, et al. Cervical screening [Internet]. Toronto, ON: Cancer Care Ontario; 2011. Available from: http://www.ontla.on.ca/library/repository/ mon/29009/332065.pdf

22. Meg McLachlin C, Mai V, Murphy J, Fung-Kee-Fung M, Chambers A, Oliver TK, et al. Ontario cervical cancer screening clinical practice guidelines. J Obstet Gynaecol Can. 2007;29(4):344-53.

23. Royal Australian College of General Practitioners. Cervical cancer. In: Guidelines for preventive activities in general practice. 8th ed. East Melbourne: Royal Australian College of General Practitioners; 2012. p. 635. ISBN 9780869063446

24. National Health and Medical Research Council. Screening to prevent cervical cancer: guidelines for the management of asymptomatic women with screen-detected abnormalities. Canberra: Commonwealth of Australia; 2005. Available from: http://www.nhmrc.gov.au/_files_nhmrc/ publications/attachments/wh39.pdf

25. University of Michigan Health System. Cancer screening [Internet]. Ann Arbor (MI): University of Michigan Health System; 2011 [updated 2014]. Available from: https://www.med.umich.edu/1info/FHP/practiceguides/adult.cancer/cancergline.pdf

26. MoyerVA, U.S. Preventive Services Task Force. Screening for cervical cancer: U.S. Preventive Services Task Force recommendation statement.Ann
Intern Med. 2012;156(12):880-91.

27. Toward Optimized Practice. Cervical cancer screening: clinical practice guideline. Edmonton, AB: topabertadoctors.org [homepage]; 2016.Available from: http://www.topalbertadoctors.org/download/587/cervical \%20cancer\%20guideline.pdf?_20180618180045

28. BC Cancer Agency. Genital tract cancers in females: human papillomavirus related cancers (cervical, vaginal \& vulvar) [Internet]. BC Cancer Agency; 2014 [updated 2016 July 15]. Available from: https://www2. gov.bc.ca/assets/gov/health/practitioner-pro/bc-guidelines/fgt_hpv.pdf

29. Massad LS, Xie X, Minkoff H, Darragh TM, D'Souza G, Sanchez-Keeland $L$, et al. Abnormal pap tests and human papillomavirus infections among HIV-infected and uninfected women who have sex with women. J Low Genit Tract Dis. 2014;18(1):50-6.

\section{CONFLITO DE INTERESSES}

As autoras declaram não ter quaisquer conflitos de interesse.

\author{
ENDEREÇO PARA CORRESPONDÊNCIA \\ Alice Jeri \\ E-mail: alicejeri@hotmail.com \\ https://orcid.org/0000-0003-1630-3156
}

Recebido em 13-03-2017

Aceite para publicação em 14-11-2018

\section{ABSTRACT}

\section{CERVICAL CANCER SCREENING IN HOMOSEXUAL PATIENTS: WHAT IS THE EVIDENCE?}

Objective: To review the evidence on the relevance of cervical cancer screening on healthy patients engaged in homosexual behavior, taking into account the risk of developing this pathology within this population.

Data sources: MEDLINE database, National Guideline Clearinghouse, National Institute for Health and Care Excellence, Canadian Medical Association Practice Guidelines Infobase, Cochrane Library, Bandolier, DARE and Trip Database.

Revision methods: Evidence based review of relevant scientific papers, published within the past five years (from 01/10/2011 to 30/09/2015), in English, Spanish and Portuguese. Combinations of the following MESH words were used: 'female homosexuality', 'mass screening', 'diagnosis', 'uterine cervical neoplasms' and 'papillomaviridae', and also the term 'cervical cancer'. The Strength of Recommendation Taxonomy of the American Family Physician was used to evaluate the Levels of Evidence (LE) and the Strength of Recommendation (SR).

Results: The search returned 88 papers, six of which met the inclusion: five clinical guidelines (one with SR A, two with SR B and two with SR C) and one prospective cohort study (LE 3). International guidelines present some limitations and inconsistencies regarding the indication of screening homosexual women, but the majority recommends their inclusion. The cohort study found abnormal results in Pap and human papillomavirus (HPV) in women who have sex with women, placing them at risk of developing cervical cancer.

Conclusions: Women engaged in homosexual behavior may be at risk of being infected by HPV and developing cervical cancer, and should therefore be included in population-based screening, with the same frequency than other women (SR B). Nonetheless, further studies are needed to reinforce this evidence. Given the lower awareness of this group on the benefits of screening, we suggest the implementation of specific educational measures in order to increase their adherence. We also suggest reviewing national guidelines so as to clarify the indication of screening in this population.

Keywords: Mass screening; Diagnosis; Uterine cervical neoplasms; Female homosexuality; Sexual behavior. 